Vidaurreta Porrero, I., Orengo Femenía, J., Gómez-Martín, A. \& De la Fe Rodríguez, C. (2021). La COVID-19 evidencia la necesidad de incrementar las competencias en economía de los estudiantes de veterinaria. Revista Electrónica Interuniversitaria de Formación del Profesorado, 24(1), 115-129.

DOI: https://doi.org/10.6018/reifop.460841

\title{
La COVID-19 evidencia la necesidad de incrementar las competencias en economía de los estudiantes de veterinaria
}

Irene Vidaurreta Porrero', Juan Orengo Femenía², Ángel Gómez-Martín ${ }^{3}$, Christian de la Fe Rodríguez ${ }^{4,5}$

${ }^{1}$ Department of Accounting and Finance, Faculty of Economics and Business, Regional, Campus of International Excellence "Campus Mare Nostrum", University of Murcia, 30100 Murcia, Spain.

${ }^{2}$ Department of Animal Production, Faculty of Veterinary Sciences, Regional, Campus of International Excellence "Campus Mare Nostrum", University of Murcia, 30100 Murcia, Spain.

${ }^{3}$ Microbiological Agents Associated with Animal Reproduction (ProVaginBio) Research Group, Department of Animal Health and Public Health, Faculty of Veterinary Sciences, University CEU Cardenal Herrera of Valencia, CEU Universities, 46113 Valencia, Spain

${ }^{4}$ Department of Animal Health, Faculty of Veterinary Sciences, Regional, Campus of International Excellence “Campus Mare Nostrum”, University of Murcia, 30100 Murcia, Spain.

${ }^{5}$ Expert on Animal Production. European Association of Establishments for Veterinary Education (EAEVE).

\section{Resumen}

La pandemia Covid-19 ha motivado la adopción de medidas excepcionales en todo el mundo, a efectos de limitar los contagios y el colapso de los sistemas sanitarios. El cierre de comercios y otras actividades consideradas no esenciales, o las limitaciones al movimiento, ha generado un impacto económico en el sector de los pequeños rumiantes, poniendo de manifiesto la necesidad del profesional veterinario de disponer de las competencias necesarias para valorar económicamente el impacto de las enfermedades en los colectivos. Este trabajo analiza, en primer lugar, la formación en economía que reciben los estudiantes del Grado de Veterinaria en las diferentes facultades españolas, comparándola con la que

Contacto:

Christian de la Fe Rodríguez, Department of Animal Health, Faculty of Veterinary Sciences, Regional, Campus of International Excellence "Campus Mare Nostrum", University of Murcia, 30100 Murcia, Spain. Phone 868887259, cdelafe@um.es. 
reciben los estudiantes en el Grado de Ingeniería Agrícola. En segundo lugar, se ha diseñado una encuesta para egresados y estudiantes de último curso de veterinaria, en referencia a este tipo de competencias, su aplicación en la sanidad animal y su repercusión en el desempeño profesional. Los datos evidencian que el tiempo empleado para adquirir las competencias en economía de los veterinarios es menor (3-6 créditos en 5 años) que el que disponen los ingenieros agrícolas (12 créditos en 4 años). Los resultados de la encuesta revelan que, si bien se registran diferencias significativas cuantitativamente entre egresados y estudiantes, ambos grupos coinciden en la escasa formación recibida para valorar el impacto de las enfermedades en los colectivos, así como en la necesidad de formarse en aspectos de gestión económica una vez finalizados sus estudios de grado para el desempeño de su labor profesional, competencias que deberían ser reforzadas en el grado. Entre los egresados, la opinión es similar, independientemente de los años de desempeño profesional.

\section{Palabras clave}

COVID-19; veterinaria; economía; grado universitario; competencias.

\section{COVID-19 highlights the need to increase health economics skills of veterinary students}

\section{Abstract}

The Covid-19 pandemic led to extreme control measures around the world aiming to halting the number of new infections. Non-essential activities closures and population confinement had an economic impact on the small ruminant sector, highlighting the need for veterinarians to have some skills to assess the economic impact of diseases on flocks. Firstly, this study analyzed the economic training received by the veterinary students at the Spanish faculties, also comparing it with the ones received by students of the agricultural engineering degree. Secondly, a survey in reference to the acquisition of this type of competences and its application for animal health was designed and applied for graduates and final-year veterinary students. The data showed that the ECTS taken to acquire the economic skills of veterinarians is less (3-6 ECTS in 5 years) than that of agricultural engineers (12 ECTS in 4 years). The results of the survey also showed that, although there are significant differences between graduates and students, both them are largely in agreement on the little training received to assess the impact of diseases, and on the need for an additional training after completing their studies. Therefore, these skills should be reinforced in the degree. Among the graduates, the opinion is similar, regardless of the years of professional experience.

\section{Key words}

COVID-19; veterinary sciences; economy; university degree; skills 


\section{Introducción}

El grado de Veterinaria cuenta, desde 1982, con planes de estudio generalistas, no de especialidad, acordes con la legislación que regula los estudios en el entorno de la Unión Europea. Deben contar con un mínimo de 5 años, cursando obligatoriamente una serie de materias que engloban los tres perfiles clásicamente considerados para la profesión (Medicina y Sanidad; Producción Animal y Economía; y Bromatología, Sanidad y Tecnología de los Alimentos), además de una serie de prácticas preprofesionales. Los requisitos para la verificación de los títulos universitarios oficiales que habilitan para el ejercicio de la profesión de Veterinario describen los contenidos mínimos que deben reunir los planes de estudio (ORDEN ECI/333/2008), cumpliendo con las directrices de la Directiva 2005/36/EC, modificada por la directiva 2013/55/EU, y su Anexo V.4.1, para el reconocimiento de la cualificación profesional por parte de los estados miembros de la Unión Europea. Además de los sistemas de evaluación nacionales, los estándares de calidad de los títulos se evalúan periódicamente por parte de la EAEVE (Asociación Europea de Establecimientos de Enseñanza Veterinaria), miembro de ENQA (Asociación Europea de Agencias de garantía de calidad), a través de un comité de expertos destinado a tal fin (Wapenaar, 2015).

Mantener la sanidad y el bienestar de las especies de abasto es un reto para la sociedad que debe ser contextualizado política, social y económicamente (Rushford et al., 2016). Así, dentro de "las competencias del día 0" de los futuros veterinarios (Day-one skills), es decir, las competencias mínimas que debería reunir un veterinario el primer día después de dejar la facultad e incorporarse a su vida profesional, se encuentra "conocer el contexto económico en el cual se desempeña la labor veterinaria", o "el conocimiento de los negocios relacionados con la cría, producción y mantenimiento de animales" (EAEVE, 2019). Estas competencias, que pueden parecer muy generales, no especifican, al menos directamente, si un veterinario debe ser capaz de calcular el impacto económico de una enfermedad en función de una serie de condicionantes normalmente relacionados con su presentación (Le Gall, 2006), si bien es bastante posible que el concepto que busquemos sea parte de la "medicina de poblaciones", es decir, el manejo global de la salud y demás factores que influyen en un colectivo. Sin embargo, tras la consulta de algunos programas docentes en varios países (datos no mostrados), la impresión es que prevalece en su totalidad el componente clínico de las enfermedades y las medidas de prevención y manejo asociadas, siendo escasas las referencias al estudio económico de las mismas.

Las consecuencias de las enfermedades de los animales pueden ser complejas y, en general, se extienden mucho mas allá del impacto inmediato sobre los productores afectados. Así, pueden considerarse pérdidas de productividad de la explotación (pérdida de producción, coste de los tratamientos, alteración del normal comportamiento de los mercados), pérdidas de renta en las actividades que utilizan los recursos animales (agricultura, energía, transporte, turismo), efectos sobre el bienestar de las poblaciones (morbilidad e incluso mortalidad, seguridad y calidad de los alimentos), costes de prevención o control (costes de producción, gasto público), o incluso la infrautilización del potencial productivo (especies animales, genética, prácticas ganaderas) (Le Gall, 2006). Trabajos recientes nos muestran que incluso cuando una enfermedad no afecta directamente a una especie, puede tener una repercusión económica importante en la misma. Por ejemplo, la Covid-19, a pesar de ser una zoonosis, no parece afectar directamente a los rumiantes (tal y como ya se evidenció con otra infección ocasionada por otro coronavirus, el MERS -Middle East Respiratory Syndrome). Sin embargo, varios autores ya alertaron al comienzo de la pandemia que ésta podía generar un efecto sanitario perjudicial en los rebaños en producción (Gortázar y De la Fuente, 2020). Aunque estos autores se referían al impacto directo de otras enfermedades sobre la gestión sanitaria de las explotaciones, una pandemia puede tener repercusiones al 
final de la cadena de producción (transformación, comercialización) en términos de empleo, ingresos del sector o precios (Le Gall, 2006). En esta línea, trabajos recientes han evidenciado el impacto económico generado por la pandemia en los rebaños de pequeños rumiantes tras el confinamiento (Vidaurreta et al., 2020a). Los datos proporcionados por los propios ganaderos mostraron un descenso de los ingresos asociado a la disminución del precio de la leche (sólo en el sector caprino) y a la caída de precio y ventas en el sector cárnico en el ovino y en el caprino.

El sector ganadero desempeña un papel significativo en el desarrollo económico de muchos países. La producción de carne y de otros productos es fuente de ingresos, de empleo y de divisas para todos los protagonistas del sector (Le Gall, 2006). Sin embargo, y a pesar de su posible efecto sobre la rentabilidad de las explotaciones, sorprendentemente, tras casi un año de pandemia, son escasos los trabajos que abordan desde el punto de vista económico las consecuencias de la pandemia. Por ello, tras los trabajos previamente realizados (Vidaurreta et al., 2020a y b), que muestran el impacto aparentemente inadvertido de la COVID-19 para los profesionales del sector de los pequeños rumiantes, en este trabajo se plantea si la formación que reciben los profesionales veterinarios en el ámbito económico es la adecuada, analizando en primer lugar los créditos que se imparten en el grado de Veterinaria relacionados con contenidos de economía, para compararlos posteriormente con los recogidos en otra titulación ligada a la producción animal. En una segunda aproximación, se ha diseñado y realizado una encuesta tanto a profesionales veterinarios del sector de los pequeños rumiantes como a estudiantes de $5^{\circ}$ curso de la titulación, al objeto de recabar información en referencia a las competencias en economía adquiridas en las Facultades y su utilidad a la hora de aplicarlas a la Sanidad y Producción Animal para el desempeño de su función profesional.

\section{Metodología}

En primer lugar, hemos realizado un análisis de la formación en el ámbito económico que se desarrolla actualmente en las Facultades de Veterinaria españolas, poniendo la atención en el número de créditos y en el curso o los cursos en los que teóricamente se adquieren dichas competencias. En este sentido, se han analizado las guías docentes de las asignaturas, evaluando la presencia de epígrafes que indiquen que se realiza el análisis económico de aspectos asociados a la sanidad y producción animal en cualquier ámbito de la profesión. Del mismo modo, en segundo lugar, hemos comparado dicha formación con los créditos que de la misma índole se imparten en el grado de ingeniería agrícola, por estar focalizada parte de su labor en los animales de abasto (gestión técnico-económica), sin abarcar nunca, evidentemente, el ámbito sanitario.

Posteriormente, hemos analizado la adquisición de las competencias en materia de economía en el título de veterinaria y su aplicación en el desarrollo de la labor profesional, al objeto de valorar la conveniencia o suficiencia de ésta. Se ha recopilado información en dos ámbitos diferentes, analizando: 1) la opinión de los profesionales veterinarios que trabajan directa o indirectamente en el sector de los pequeños rumiantes, considerando dos grupos de estudio (más de 20 años de experiencia y menos de 20 años de experiencia), y 2) la opinión de los estudiantes de $5^{\circ}$ del Grado de Veterinaria de diferentes Facultades españolas.

Para conseguir este último propósito, se ha diseñado una encuesta con el objetivo de conocer las diversas opiniones, valorando de 1 a 5 (el grado de conformidad en una escala de Likert creciente) cada uno de los siguientes ítems: 
1. Los contenidos de economía que aprendiste en la carrera serán o han sido (en función del grupo) útiles en el desarrollo de la labor profesional.

2. Tendrás o has tenido (en función del grupo) que formarte externamente en aspectos económicos para desarrollar tu labor profesional.

3. En la carrera me enseñaron a calcular el impacto económico de la sanidad (o una enfermedad concreta) en un colectivo.

4. En base a tu experiencia, sería interesante incrementar el tiempo dedicado a valorizar factores como las enfermedades en el grado de veterinaria.

5. Incrementar el tiempo de aprendizaje del impacto económico de la sanidad animal me haría mejor profesional.

Las preguntas 1 y 2 valoran esencialmente el aspecto formativo tanto a nivel de facultad como con posterioridad a la finalización de los estudios universitarios. Las preguntas 3-5, conforman un bloque asociado a definir las competencias adquiridas a la hora de valorar el impacto económico de una enfermedad en los colectivos y su posible repercusión en el desempeño de la actividad profesional.

A nivel analítico, se realizó un estudio descriptivo de los participantes en función del perfil del encuestado (estudiante vs. egresado) y la experiencia profesional. Asimismo, se calcularon los principales estadísticos de posición central (media, mediana, moda) y dispersión (desviación estándar), junto con los intervalos de confianza al 95\% para los promedios muestrales de las respuestas a cada pregunta. Posteriormente, los datos se analizaron teniendo en cuenta la influencia de las variables o factores de interés (perfil del encuestado y experiencia profesional) mediante test o pruebas de indepencia chi-cuadrado $\left(\chi^{2}\right)$. Por otro lado, teniendo en cuenta que las respuestas eran evaluadas en una escala ordinal, se recurrió al coeficiente de correlación no paramétrico de Spearman o rho de Spearman $(\rho)$ para analizar el grado de asociación y dirección de la relación entre las respuestas/preguntas.

\section{Resultados}

\section{Distribución de créditos en las Facultades de veterinaria}

Se han consultado un total de 12 planes de estudio ( 9 de universidades públicas y 3 privadas). Todos ellos incluyen los contenidos básicos asociados a la economía y a la gestión de las empresas, siguiendo las directrices de la legislación europea que regula la profesión. Aunque es complejo saberlo con precisión, el número de créditos que se destina a tal fin oscila entre 3 y 6 créditos según el plan de estudios de cada Facultad (Tabla 1), lo cual corresponde al 1-2\% del total de créditos del Grado. En algunos casos, parte de los créditos asignados son compartidos con otras competencias o contenidos (generalmente agronomía o análisis y gestión de datos), mientras en otros planes de estudio, no es posible determinar con exactitud los créditos impartidos o el número de horas o bloques que se dedican a la adquisición de las competencias en este ámbito. Por ejemplo, algunas Facultades disponen de planes de estudio donde se agrupan todos los aspectos de gestión, sanidad o producción de cada especie de abasto en una misma asignatura, lo que hace complejo conocer específicamente el número de horas que se dedica a cada aspecto, o las diferencias entre asignaturas que versan sobre diferentes especies. Evidentemente, esto no quiere decir en ningún caso que esos temas no se aborden transversalmente en el currículo. 
Como puede observarse en la Tabla 1, los contenidos no se imparten en el mismo curso en los centros analizados. De hecho, aproximadamente en la mitad de ellos se imparte entre $3^{\circ}$ y $4^{\circ}$ curso (e incluso en $5^{\circ}$ ), mientras que en otros planes de estudio son contenidos que se imparten principalmente entre $1^{\circ}$ y $2^{\circ}$ curso. Esta diversidad en la ubicación de contenidos responde principalmente a que las competencias en economía tales como "Marketing y gestión empresarial de ámbito general y veterinario" y "Economía del proceso productivo y comercialización. Desarrollo sostenible" aparecen vinculadas a los módulos de Formación Básica Común y Producción Animal, respectivamente, de acuerdo con la ficha técnica del título de Grado en Veterinaria (ORDEN ECI/333/2008).

Tabla 1. Distribución de créditos por Facultad asociado a contenidos de economía

\begin{tabular}{|c|c|c|c|}
\hline Facultad & Créditos & Curso/s & Asignatura/s \\
\hline Universidad de Murcia & $6 *+3$ & $1^{0}-4^{0}$ & 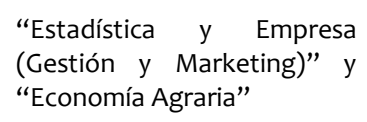 \\
\hline Universidad de Las Palmas GC & 3 & $4^{\circ}$ & $\begin{array}{l}\text { "Economía } \\
\text { Comercialización } \\
\text { Agroalimentaria" }\end{array}$ \\
\hline Universidad Complutense Madrid & $6 *$ & $2^{\circ}$ & $\begin{array}{l}\text { "Bases de la producción } \\
\text { Animal II: Agronomía, } \\
\text { Economía y Gestión de } \\
\text { Empresas Veterinarias" }\end{array}$ \\
\hline Universidad de Santiago & 3 & $2^{\circ}$ & “Economía Agraria” \\
\hline Universidad de Córdoba & $6^{*}+3$ & $1^{0}-5^{0}$ & $\begin{array}{l}\text { "Análisis de Datos y Gestión } \\
\text { Veterinaria" y "Economía de } \\
\text { la Producción Ganadera" }\end{array}$ \\
\hline Universidad de Extremadura & $6 *$ & $1^{\circ}$ & $\begin{array}{l}\text { "Agronomía y Economía } \\
\text { Agraria" }\end{array}$ \\
\hline Universidad Autónoma Barcelona & $6^{*}+3^{*}$ & $1^{0}-5^{\circ}$ & $\begin{array}{lrr} & \text { "Agronomía y } & \text { Economía } \\
\text { Agraria" y } & \text { "Ética y y } \\
\text { Legislación. } & \text { Gestión } \\
\text { Empresarial" } & & \end{array}$ \\
\hline Universidad de León & 6 & $3^{\circ}$ & $\begin{array}{l}\text { "Economía Agraria u } \\
\text { Gestión" }\end{array}$ \\
\hline Universidad de Zaragoza & 6 & $1^{\circ}$ & “Economía y Empresa” \\
\hline Universidad Cardenal Herrera** & ¿? & $3^{\circ}-4^{\circ}$ & $\begin{array}{l}\text { Integración en diversas } \\
\text { asignaturas }\end{array}$ \\
\hline Universidad Alfonso X** & $6 *$ & $2^{\circ}$ & $\begin{array}{lll}\text { "Etnología } & \text { y } & \text { gestión } \\
\text { empresarial } & \text { en } & \text { ámbito } \\
\text { veterinario" } & & \end{array}$ \\
\hline Universidad Católica** & 6 & $3^{\circ}$ & $\begin{array}{l}\text { "Economía y Empresa en el } \\
\text { ámbito veterinario" }\end{array}$ \\
\hline
\end{tabular}

*Los créditos incluyen otros contenidos. **Universidades privadas. 


\section{Comparación de créditos en los Grados de Ingeniería Agrícola}

Un total de 48 centros imparten alguna titulación donde se incluye la ingeniería agrícola, agroalimentaria o rural. Es una titulación de 240 créditos que se imparte en 4 años. En algunos casos se ofrecen con dobles titulaciones, agrupándose, entre otras, con ciencias ambientales. Se han consultado los 5 planes de estudio de las facultades mejor clasificadas en función del Ranking del periódico El Mundo para el año 2019 (Universidad Politécnica de Madrid, Universidad de Córdoba, Universidad de Lérida, Universidad de Almería y Universidad de Sevilla). En todos los planes de estudio consultados se incluyen contenidos asociados a la economía en dos asignaturas principalmente de primer y segundo curso que, en conjunto, representan aproximadamente 12 créditos del plan de estudios, lo cual corresponde al $5 \%$ del total de créditos del grado. En los casos considerados, los contenidos se agrupan al menos en dos asignaturas obligatorias de 6 créditos, en apariencia bastante homogéneas entre los centros.

\section{Análisis de la encuesta a estudiantes y profesionales veterinarios}

\section{Descripción de la muestra}

En este apartado se describen las características de la muestra respecto a las variables de identificación, para destacar los aspectos más importantes (Gráfico 1). Se han recibido un total de 243 encuestas, incluyendo un total de 109 egresados que trabajan directa o indirectamente con el sector de los pequeños rumiantes (45\% de la muestra) y que han desarrollado sus estudios en 11 de las 13 facultades españolas. Del mismo modo, se ha recibido la respuesta de un total de 134 estudiantes de $5^{\circ}$ curso de las Facultades de Veterinaria en España (55\%). En referencia a la experiencia profesional, el $50 \%$ de los participantes tiene una experiencia menor o mayor a 20 años (55 y 54 egresados, respectivamente). Dentro de las dos clases establecidas para la experiencia profesional, existía también un equilibrio cuando las categorías establecidas eran, a su vez, subdivididas en menos de 15 años y más de 30, respectivamente (con proporciones entre 22 y $28 \%$ por categoría). Respecto al género de los participantes y, a pesar de que no ha sido considerado como un factor en el análisis, hubo un total de 150 y 93 mujeres y hombres; si bien la proporción de mujeres era mayor entre los estudiantes (más del 80\%), y dicho porcentaje se reducía significativamente con la mayor experiencia profesional de los egresados.

Gráfico 1. Descripción y características de los participantes en la encuesta $(n=243)$.

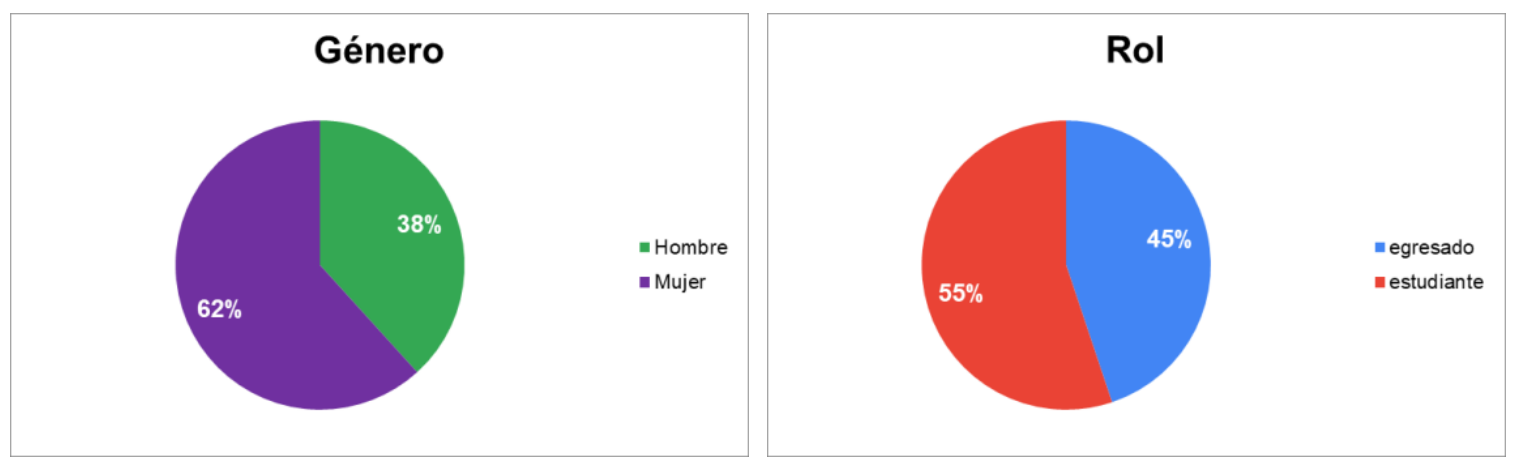




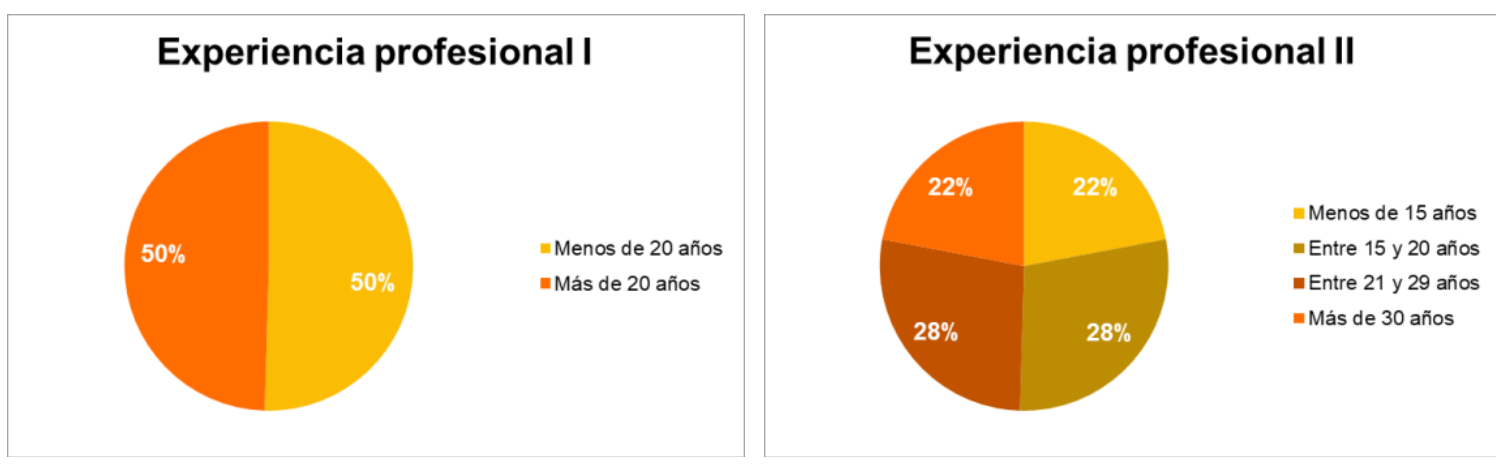

\section{Valoración global}

La tabla 2 muestra los resultados medios y dispersión para cada una de las preguntas planteadas.

Tabla 2. Estadísticos descriptivos para las respuestas de la encuesta en una escala creciente de 1 a 5 puntos.

\begin{tabular}{cccccc}
\hline Estadístico & Pregunta 1 & Pregunta 2 & Pregunta 3 & Pregunta 4 & Pregunta 5 \\
\hline $\begin{array}{c}\text { Media } \\
\text { Intervalo de } \\
\begin{array}{c}\text { confianza } \\
\quad(95 \%)\end{array}\end{array}$ & 2,31 & 4,07 & 2,03 & 4,12 & 4,04 \\
Mediana & $2,16-2,46$ & $3,93-4,21$ & $1,88-2,18$ & $4,00-4,21$ & $3,90-4,18$ \\
Moda & 2 & 4 & 2 & 4 & 4 \\
$\begin{array}{c}\text { Desviación } \\
\text { estándar }\end{array}$ & 2 & 5 & 1 & 5 & 5 \\
\hline
\end{tabular}

Los valores de los estadísticos de posición central de la tabla 2 en relación a la pregunta 1 reflejan cierto grado de disconformidad de los participantes sobre los contenidos de economía recibidos en la Facultad (con valores en torno a 2 sobre 5). Respecto a la distribución de las respuestas, un total de 154 encuestados $(63,45 \%)$ no considera que los contenidos de economía aprendidos en la carrera le hayan sido o serán de utilidad en el desempeño de su actividad profesional ( 1 o 2 puntos en una escala de 5), lo cual contrasta con el $21 \%$ que considera que está de acuerdo con su utilidad ( 51 encuestados), y el 15,6\% (38 encuestados) que opinan que han sido o serán muy útiles (4 o 5 puntos). Curiosamente, estos datos, contrastan con las respuestas a la pregunta 2, con un valor medio y moda de 4 y 5 puntos, respectivamente, en la que la mayor parte de los encuestados (219 encuestados, 90\%) manifiestan que tendrán o han tenido que formarse externamente en esta materia para desarrollar su actividad profesional. Nuevamente, y tal como evidencia las respuestas a la pregunta 3, cuyo grado de conformidad medio es de 2 puntos sobre una escala de 5 , un elevado porcentaje de los encuestados considera que no ha adquirido las competencias necesarias para valorar económicamente el impacto de una enfermedad en un colectivo (70\%, 170 encuestados). En este sentido, las respuestas de los participantes a las últimas preguntas (con valores medios y moda de 4 y 5 puntos respectivamente), muestra mayoritariamente la necesidad de incrementar la adquisición de este tipo de competencias en el grado de veterinaria (pregunta 4: 91\%, 221 encuestados), y manifiesta que la mejor 
adquisición de esas competencias le repercutiría positivamente en el desempeño de su labor profesional (pregunta 5: 90,1\%, 219 encuestados).

\section{Influencia de los factores: perfil del encuestado y experiencia profesional}

Los datos obtenidos, reflejan la existencia de diferencias significativas a la hora de responder en función del perfil del encuestado, bien sea egresado o estudiante del último año, para prácticamente la totalidad de las preguntas planteadas. Así, podemos observar que las respuestas de los estudiantes en el bloque 1 de formación (gráfico 2), que engloba las 2 primeras preguntas, registran valores medios superiores respecto a los egresados. No obstante, ambos colectivos coinciden en la valoración de los contenidos económicos recibidos en la Facultad y en la necesidad de formarse con posterioridad, aspecto este último que registra valores medios más altos en los estudiantes de último año respecto a los egresados. Asimismo, los egresados con menor experiencia profesional son los que muestran significativamente el mayor grado de disconformidad respecto a la formación recibida en la Facultad, con un valor medio de 1,65 puntos.

En referencia al segundo bloque (gráfico 3) y a la valoración económica de las enfermedades, las respuestas de los egresados sugieren que han recibido una formación menor en este ámbito respecto a los estudiantes de último curso, y son ellos, sin embargo, los que cuantitativamente reflejan de un modo superior la necesidad de incrementar la adquisición de estas competencias en las facultades, por la repercusión positiva que tiene para el desempeño de la labor profesional. Considerando la experiencia profesional de los egresados, la opinión de los mismos refleja que no hay diferencias a la hora de contestar las preguntas planteadas, más allá de algunas tendencias, que no obstante no llegan a ser significativas. 
Gráfico 2. Histogramas de frecuencias absolutas por pregunta en el bloque sobre formación en economía, con tests de asociación o independencia chi-cuadrado $\left(\chi^{2}\right)$ según el perfil del encuestado o experiencia profesional.
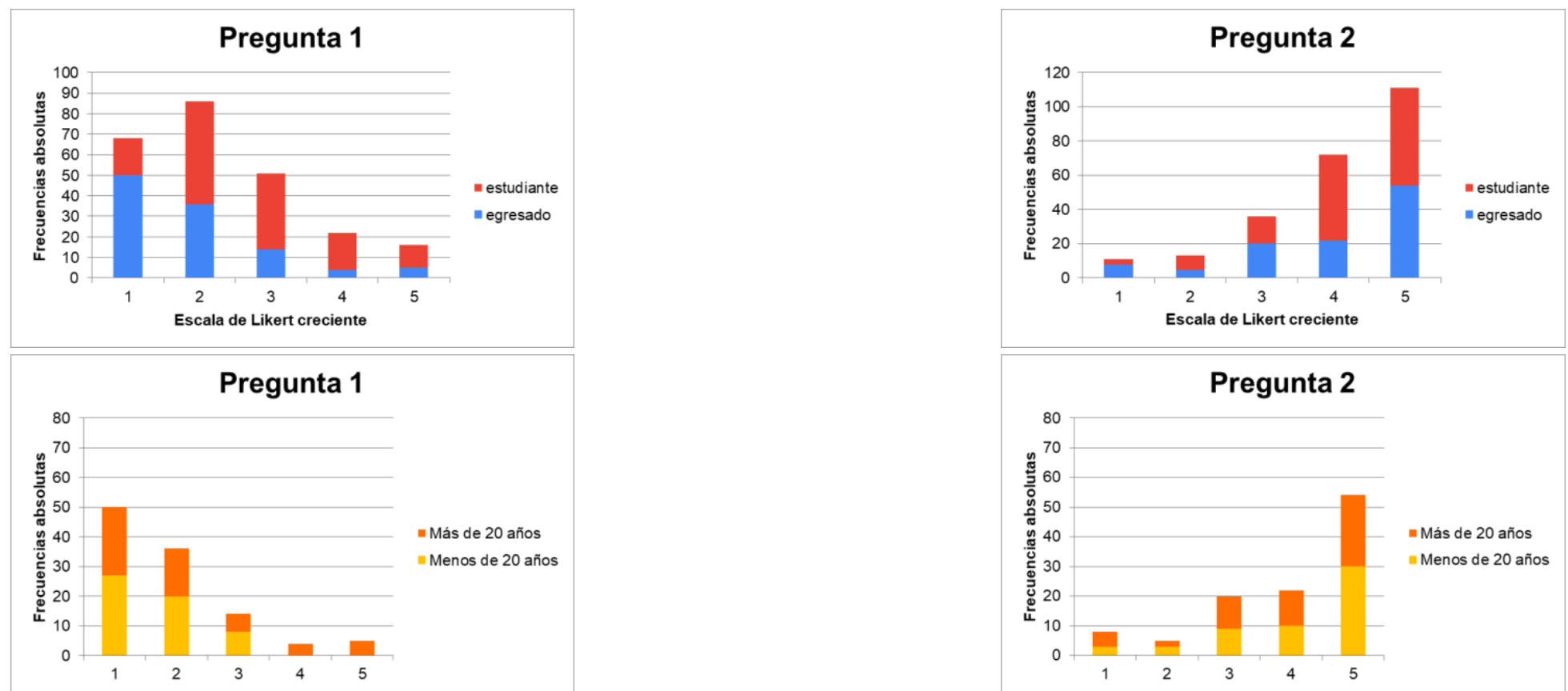

$\chi^{2} \sim p$-values

\begin{tabular}{lccc} 
Factor & Pregunta 1 & Factor & Pregunta 2 \\
\cline { 2 - 2 } Perfil del encuestado & $<0.001$ & Perfil del encuestado & 0.018 \\
Experiencia & 0.032 & Experiencia & 0.829 \\
\hline
\end{tabular}


Gráfico 3. Histogramas de frecuencias absolutas por pregunta en el bloque sobre valoración del impacto económico de las enfermedades, con tests de asociación o independencia chi-cuadrado $\left(\chi^{2}\right)$ según el rol o experiencia profesional.

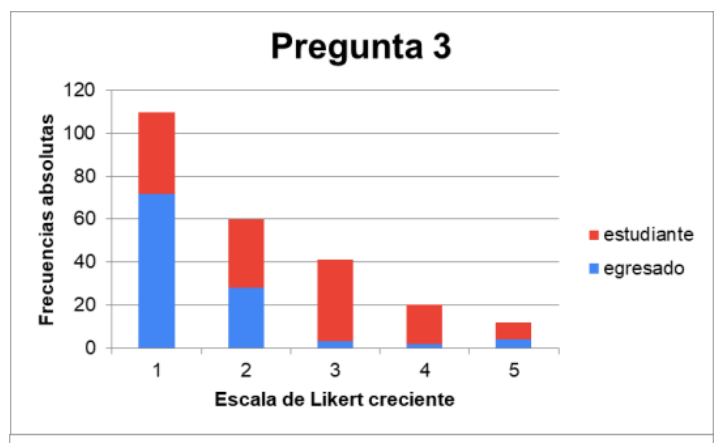

Pregunta 3
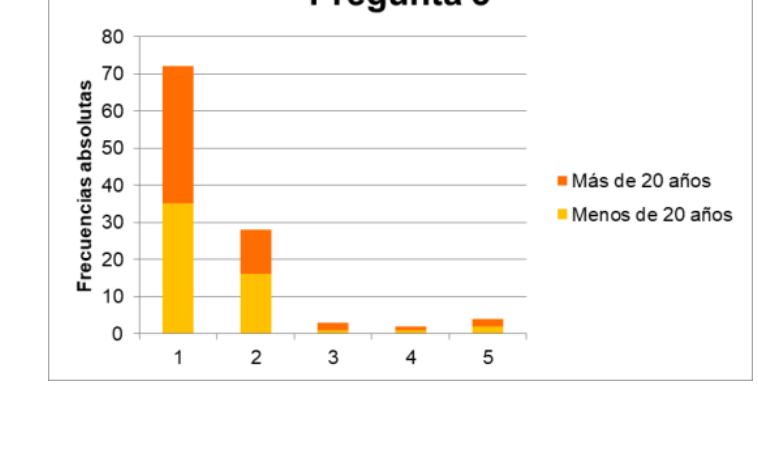

Factor

Rol

Experiencia

\begin{tabular}{c} 
Pregunta 3 \\
\hline$<0.001$ \\
0.935
\end{tabular}

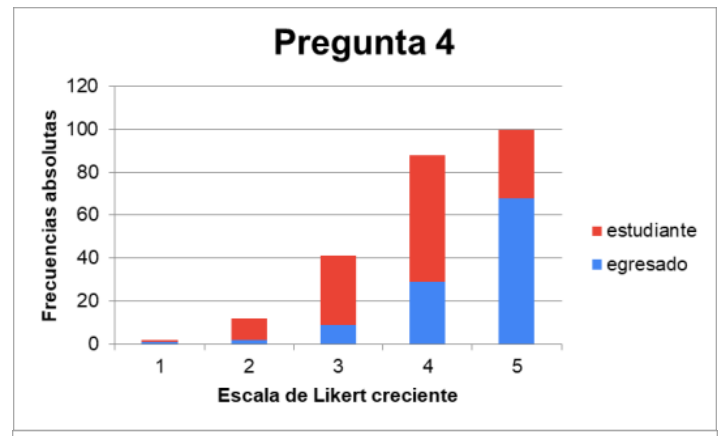

Pregunta 4

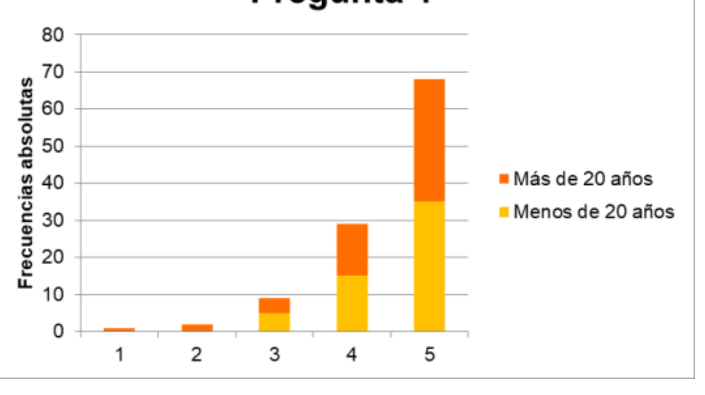

$\chi^{2} \sim p$-values

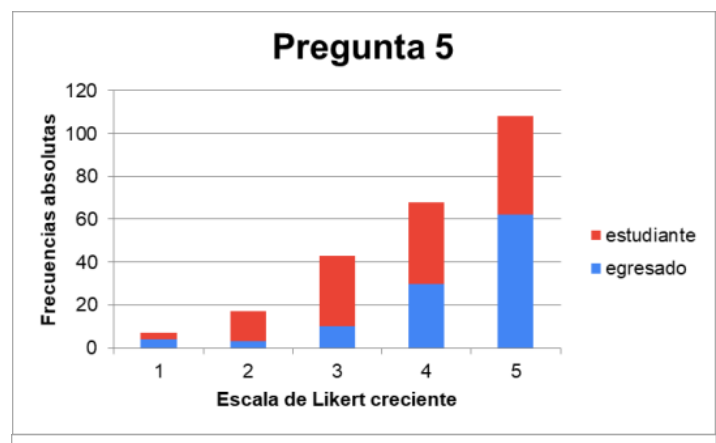

Pregunta 5

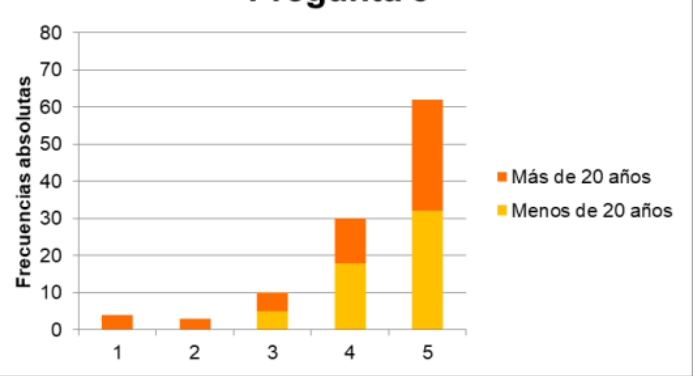

\begin{abstract}
Factor
\end{abstract}
Rol

Experiencia

Pregunta 4
$<0.001$
0.404

Factor

Pregunta 5

Rol

$<0.001$

Experiencia

0.072 


\section{Correlaciones entre las respuestas}

El análisis de los datos mostró una correlación positiva y relativamente fuerte $(>0,5)$ entre las preguntas 4 y 5 en las que los encuestados vincularon la necesidad de incrementar la adquisición de competencias en economía con el mejor desempeño de la actividad profesional. Otras relaciones significativas y positivas, pero de menor magnitud (en torno a 0,3-0.4), fueron observadas entre algunas cuestiones como la formación adquirida en la facultad y la escasa formación para analizar el impacto económico de las enfermedades (pregunta 1 y 3), o la necesidad de formarse externamente y de incrementar los contenidos en este ámbito que se imparten en las facultades (pregunta 2 y 4) y, a su vez, permitir un mejor ejercicio de la actividad profesional (pregunta 2 y 5 ). El resto de asociaciones fueron no significativas, o más débiles $(<0,3)$ y de sentido contrario. Así, la menor capacidad para saber calcular económicamente como afecta una infección o una enfermedad a un colectivo, apunta a la necesidad de incrementar la adquisición de estas competencias en los estudios de grado (preguntas 3 y 4 ).

Tabla 3. Matriz de correlaciones entre respuestas ( $\rho$, Rho de Spearman).

\begin{tabular}{lrrrr}
\hline & Pregunta 2 & Pregunta 3 & Pregunta 4 & Pregunta 5 \\
\hline Pregunta 1 &,- 023 &, $377^{* *}$ &,- 106 &, 005 \\
Pregunta 2 & &,- 012 &, $272^{* *}$ &, $323^{* *}$ \\
Pregunta 3 & &,$- 251^{* *}$ &,$- 130^{*}$ \\
$\begin{array}{l}\text { Pregunta 4 } \\
\text { "**” and “**” indican que la correlación es significativa en el nivel 0.05 y } 0.01 \text { (bilateral), } \\
\text { respectivamente. }\end{array}$
\end{tabular}

\section{Discusión y conclusiones}

Aunque la Sanidad y Producción animal sea una labor exclusiva de la profesión veterinaria, dicha actividad no puede menoscabar la necesidad de manejar y realizar una gestión integral de las explotaciones, para lo cual es necesario utilizar una serie de herramientas básicas de gestión técnico-económica. La sanidad y el bienestar de los animales tiene un impacto directo en los ingresos de las explotaciones (Mclnerney et al., 1992), y recibir una educación que abarque todos los aspectos asociados al manejo económico de las explotaciones y la influencia de la Sanidad es un desafío, teniendo en cuenta que, en muchos centros de todo el mundo, la educación se ha focalizado en los aspectos clínicos que afectan a perros y gatos (Rushton et al., 2016).

El análisis de los planes de estudio vigentes en las Facultades españolas de Veterinaria, revela, en primer lugar, que todas ellas cumplen con la normativa vigente en cuanto a la impartición de créditos que cubran las competencias en esta materia (ORDEN ECI/333/2008; EAEVE, 2019), si bien es difícil evaluar en los programas docentes su aplicación directa al estudio de las enfermedades o al cálculo del impacto económico de las mismas. Es evidente que estas competencias pueden adquirirse también al cursar asignaturas de las áreas de Sanidad Animal, Producción Animal, o incluso Medicina y Cirugía, si bien los contenidos que se imparten al respecto suelen estar centrados en describir la importancia de cada enfermedad en su contexto productivo, sin entrar en detalles económicos.

Desde un punto de vista más general, la comparación de los créditos referentes a contenidos económicos impartidos en el Grado de Veterinaria con el Grado de ingeniería agrícola, también ligado a las actividades ganaderas, evidencia que la economía dispone de 
un mayor ámbito de estudio en esta última titulación, si bien, evidentemente, no está orientada nunca al ámbito sanitario. Algunos autores sugieren que ya desde el comienzo de los estudios de Veterinaria se busca fortalecer la biología, la química y la física, mientras se da por suficiente el nivel de conocimientos y habilidades en matemáticas que poseen los estudiantes que ingresan en la mayoría de las facultades españolas (Climént, 2014). Volviendo al ámbito sanitario, es evidente que una enfermedad genera un coste directo, pero también un coste de oportunidad (Mclnerney et al., 1992). Realizar un análisis costebeneficio permite valorar la viabilidad de la inversión y el beneficio neto de las intervenciones veterinarias, lo cual ayuda al propietario a adoptar una decisión al respecto de su realización (Rushford, 2009). En el contexto socioeconómico actual, con el manejo cada vez más tecnificado y la gestión empresarial de muchas ganaderías (incluyendo la de los pequeños rumiantes), y la presencia de factores externos que pueden motivar el incremento de la volatilidad de los precios en el mercado agrario (FAO, 2010), los docentes del grado de Veterinaria deberíamos valorar cómo reforzar las competencias de nuestros futuros egresados en este tipo de competencias.

Las competencias del veterinario deben desarrollarse bajo unas condiciones concretas, que incluyen las económicas (ORDEN ECl/333/2008), lo cual tiene implicaciones directas en diversas asignaturas (Climént, 2014). La Covid-19 ha motivado una disminución inesperada de los ingresos de muchos sectores (Rodríguez-Antón y Alonso-Almeida, 2020), ocasionando un serio perjuicio económico en muchas empresas ganaderas del sector de los pequeños rumiantes (Vidaurreta et al., 2020a; 2020b). En esta línea, la necesidad, cada vez mayor, de que los técnicos veterinarios seamos a la par gestores integrales de la sanidad, y seamos capaces de traducir a "números" los efectos que ocasiona una enfermedad, o los beneficios que entraña la lucha frente a ella, es, a juicio de los autores, fundamental en el contexto actual (Climent, 2014); tal y como parece deducirse de los resultados obtenidos en el presente estudio a partir de las respuestas de los egresados y de los estudiantes de último curso, futuros veterinarios. Reconocen que esos contenidos en economía son fundamentales para el desempeño profesional, si bien, coinciden también a la hora de requerir o haber requerido de una formación extra fuera de la Facultad para poder desempeñar su labor profesional. Aunque los estudiantes valoran cuantitativamente mejor las competencias adquiridas en la Facultad respecto a los egresados, en líneas generales no consideran que la formación adquirida en las facultades sea suficiente, una impresión que se acrecienta cuando analizamos las competencias adquiridas para evaluar el impacto económico de la sanidad en las explotaciones. La respuesta de muchos técnicos, en ocasiones con más de 20 años de experiencia, no deja lugar a dudas en este sentido. Del mismo modo, indican que conocer mejor los aspectos económicos aplicados al impacto sanitario de una infección les haría mejores profesionales. El colectivo de estudiantes encuestados considera que ha adquirido más competencias a la hora de valorizar el impacto de las enfermedades que sus predecesores, si bien coinciden con ellos en la necesidad de incrementar los contenidos de esta índole, que les serían de utilidad para ser, o considerarse, mejores profesionales.

La complejidad de los efectos inducidos por una enfermedad es tal que ni siquiera es posible evaluar con precisión los efectos económicos y sociales. En España, conocemos perfectamente el impacto de determinadas enfermedades que, por ejemplo, afectan a los pequeños rumiantes. La brucelosis, la tuberculosis, la lentivirosis, la agalaxia contagiosa o la paratuberculosis generan pérdidas diarias en nuestras explotaciones $\mathrm{y}$, sin embargo, son escasas las publicaciones científicas que valoran cuantitativamente lo que estas infecciones suponen y han supuesto en nuestras empresas. Conocer el impacto de las enfermedades es fundamental incluso para la relación con los propietarios, pues facilita acercar a éstos las consecuencias de una determinada enfermedad en su ganadería (Rushford et al., 2016). Sin 
embargo, siendo fundamental la influencia de la Sanidad en los resultados finales del balance coste-beneficio de estas empresas (Rushford, 2009), sólo dos planes de estudio de las universidades españolas mencionan específicamente cuestiones como "los aspectos económicos de la calidad y la sanidad" o "las bases de la economía de la sanidad animal y el análisis de los proyectos sanitarios", lo cual, no es óbice, como indicamos anteriormente, para que los contenidos se aborden transversalmente en diferentes asignaturas. Aunque así fuera, no lo perciben así los egresados; ni los más veteranos, herederos del antiguo sistema de especializaciones, ni los más jóvenes, recién egresados o con una experiencia menor. La solución puede pasar por no impartir más créditos, un aspecto siempre complejo en las titulaciones, sino por reorientar algunos de los temas abordados en las asignaturas directamente implicadas, y también porque otras asignaturas de las áreas de conocimiento antes mencionadas, como Sanidad y Producción Animal, "recojan el guante" e impartan transversalmente algunos de esos contenidos. En este sentido, la ORDEN ECI/333/2008 es ciertamente ambigua, pues la única mención económica respecto a las competencias a adquirir dentro del bloque de Ciencias clínicas y Sanidad menciona la "Promoción de la salud en los colectivos animales, incluidos los animales silvestres, con el fin de obtener el máximo rendimiento económico de una forma social, ética y sanitariamente aceptables". Es, ciertamente, interpretable.

En conclusión, es evidente que los animales de abasto se explotan con fines económicos, y la rentabilidad de las empresas ganaderas es fundamental. Tomar decisiones sanitarias evaluando el coste y los beneficios de una acción, facilita la adopción de decisiones más racionales, para lo cual se requiere la adquisición de determinadas competencias. Los resultados de este trabajo, llevado a cabo tras el análisis del impacto económico de la COVID-19 en el sector de los pequeños rumiantes (Vidaurreta et al., 2020a, 2020b), sugieren que es necesario incrementar las competencias que nuestros futuros egresados tienen en el ámbito de la economía aplicada a su día a día como técnicos y garantes de la gestión sanitaria integral de las explotaciones. Disponer de más herramientas económicas que complementen la labor clínica y zootécnica del veterinario, debe permitir abordar de un modo más global todos los problemas que genera la Sanidad en las explotaciones.

\section{Referencias}

Climént, J.B. (2014). Tipología de las competencias en educación veterinaria. Revista de Investigaciones Veterinarias del Perú 25(2), 293-316.

DIRECTIVA 2005/36/CE DEL PARLAMENTO EUROPEO Y DEL CONSEJO de 7 de septiembre de 2005. relativa al reconocimiento de cualificaciones profesionales. https://www.boe.es/doue/2005/255/L00022-00142.pdf.

European System of Evaluation of Veterinary Training (2019). Manual of Standard Operating Procedure.https://www.eaeve.org/fileadmin/downloads/SOP/ESEVT_SOP_2019_ado pted_by_the_32nd_GA_in_Zagreb_on_30_May_2019_Updated_Annexes_16a__16 b.pdf.

Food and Agriculture Organization of the United Nations (FAO) (2010). Price Volatility in Agricultural Markets: Evidence, Impact on Food Security and Policy Responses. Economic and Social Perspectives; Policy Brief No. 12; FAO Economic and Social Development Department: Rome, Italy.

Gortázar, C., and de la Fuente, J. (2020). COVID-19 is likely to impact animal health. Preventive Veterinary Medicine, 180, 105030. 
Le Gall, F.G. (2006). Justificación económica y social de las inversiones en materia de sanidad animal y zoonosis. Conf. OIE 2006, 71-86.

Libro Blanco Título de Grado de Veterinaria. http://www.aneca.es/var/media/150400/libroblanco jun05_veterinaria.pdf

McInerney, J. P., Howe, K. S., and Schepers, J.A. (1992). A framework for the economic analysis of disease in farm livestock. Preventive Veterinary Medicine, 13(2), 137-154.

ORDEN ECI/333/2008, de 13 de febrero, por la que se establecen los requisitos para la verificación de los títulos universitarios oficiales que habiliten para el ejercicio de la profesión de Veterinario. BOE núm. 40 de 15 febrero 2008.

Rodríguez-Antón, J.M., and Alonso-Almeida, M.M. (2020). COVID-19 Impacts and Recovery Strategies: The Case of the Hospitality Industry in Spain. Sustainability, 12, 8599.

Rushton, J. (2009). Economics of Animal Health and Production. UK: CABI.

Rushton, J., Jackson, E., Häsler, B., and Redmond, E.F. (2016). Economics of production animal health. OIE, Veterinary Education Conference.

Vidaurreta, I., de la Fe, C., Orengo, J., Gómez-Martín, A., and Benito, B. (2020). Short-term economic impact of COVID-19 on Spanish small ruminant flocks. Animals, 10(8), 1357.

Vidaurreta, I., Orengo, J., de la Fe, C., González, J. Ma., Gómez-Martín, A., and Benito, B. (2020). "Price fluctuation, Protected Geographical Indications and Employment in the Spanish Small Ruminant sector during the COVID-19 crisis", Animals, 10(12), 2221.

Wapenaar, W. (2015). Interview to Dr Ana M. Bravo del Moral. REDU. Revista de Docencia Universitaria, 13(3), 207-210. 